\title{
LA DURACIÓN SILÁBICA Y CLASIFICACIÓN RÍTMICA DEL INGLÉS
}

\author{
María Heliodora Cuenca Villarín
}

\section{Introducción.}

Tradicionalmente las lenguas han sido clasificadas rítmicamente como lenguas de compás acentual ("stress-timed") y lenguas de compás silábico ("syllable-timed") 1. En las lenguas del primer tipo el período entre acentos será aproximadamente isócrono, sin atender al número de sílabas inacentuadas que lo conforman. Por el contrario, en las lenguas del segundo tipo, el intervalo entre acentos variará en función del número de sílabas inacentuadas que comprenda, pues la duración de la sílaba es estable. Sin embargo, a causa de la falta de datos acústicos que corroboren tales hipótesis en la producción del habla, la isocronía actualmente es concebida más bien como un fenómeno de la percepción ${ }^{2} \mathrm{o}$, como escribe Giegerich (1985), una realidad fonológica, para quien el pie acentual (unidad que se extiende desde el arranque de una sílaba tónica hasta el arranque de la siguiente tónica) representa una unidad de compás fonológico.

Por consiguiente, la clasificación rítmica de las lenguas ha ido adquiriendo una orientación gradual y en su caracterización rítmica se adopta una serie de criterios fonéticos y fonológicos que incluyen la estructura y duración silábicas. En efecto, las lenguas de compás acentual se caracterizarán por una mayor variedad de tipos silábicos, de estructura más compleja ${ }^{3}$, mientras que en las lenguas de compás silábico predominarán las sílabas abiertas. Por otro lado, la estructura silábica y el acento muestran una fuerte tendencia a reforzarse mutuamente en las lenguas de compás acentual, es decir, las sílabas complejas suelen ser acentuadas y las sílabas simples inacentuadas. ${ }^{4}$

Bertinetto (1989), Fauré et al. (1980), Nakatani et al. (1981) o Fant et al. (1991) forman parte de una línea de investigadores que opinan que la diferencia fundamental entre las lenguas de compás acentual y las lenguas de compás silábico puede encontrarse en la mayor o menor fuerza del contraste entre sílabas acentuadas e inacentuadas.

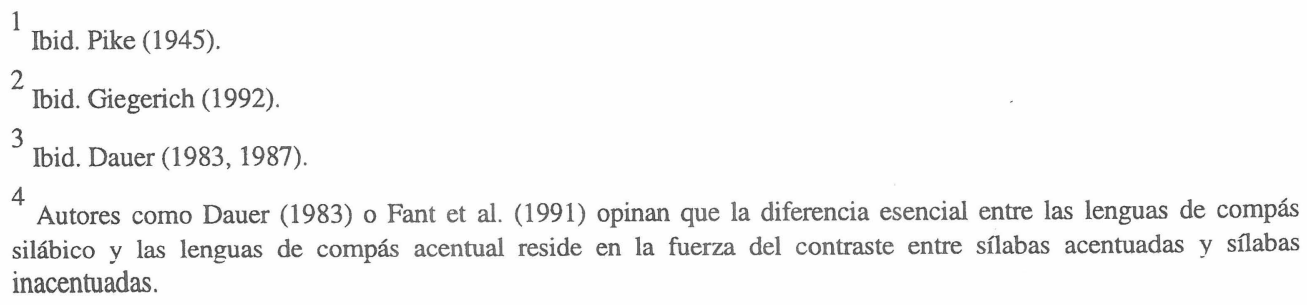


La compensación intersilábica, que recibe el nombre técnico de "compensatory shortening" 5 , es un término que puede hacer referencia, indistintamente, a la compresión de la sílaba acentuada, o de su núcleo, en función del número de sílabas inacentuadas adyacentes, y al acortamiento del núcleo silábico o de la sílaba acentuada de una palabra a causa del número de sílabas que la constituyen. Según Bertinetto (1989), las lenguas de compás silábico parecen resistirse a la compresión ${ }^{6}$, mientras que las lenguas de compás acentual la favorecen. ${ }^{7}$

Por último, en 1966 Delattre analizó la duración en sílabas del español, del francés, del inglés y del alemán, considerando que la duración relativa de las sílabas en habla fluida determina su valor perceptual, teniéndose en cuenta para ello: la condición de la sílaba según el acento ${ }^{8}$, la posición de la sílaba en el grupo de sentido - final o no final - ${ }^{9}$ y el tipo de sílaba, abierto o cerrado. ${ }^{10}$

En este trabajo, pues, adoptaremos un enfoque puramente fonético y desde la producción del habla, evaluando a través del análisis de un corpus de frases marco el efecto en la duración de la sílaba en inglés de factores cuya pertinencia ha sido establecida de forma recurrente en ésta u otras lenguas en la literatura especializada, para proceder seguidamente a la caracterización rítmica de dicha lengua según aquellos criterios fonéticos y fonológicos relacionados con la sílaba propuestos en la revisión de la tradicional tipología rítmica.

\section{Protocolo experimental.}

Se ha analizado un corpus de frases marco ("Say the word again") consistente en un cuerpo de 452 palabras con distinto número de sílabas y patrón acentual, creándose de este modo contextos homogéneos para todas ellas, y han sido presentadas en tarjetas al azar a un informante nativo británico, culto y representante de la variedad de inglés R.P., para su lectura a una velocidad normal.

Hemos tratado de asignar un indicador de velocidad a todas las frases para corregir las posibles desviaciones que se produjesen en las duraciones por la mayor o menor velocidad de producción. Para ello, se ha medido la duración de la única parte fija en las frases marco

\footnotetext{
5 Ibid. Bertinetto (1989).

6 Ibid. Hoequist (1983a, 1983b), Vayra et al. (1984), Strangert (1985), y Engstrand (1987).

7 Ibid. Nooteboom (1972), Lindblom (1975), Fowler (1977), Hoequist (1983a, 1983b), y Strangert (1985).

8 Otros autores como Dauer (1983), Toledo (1988), Cantín y Ríos (1991) y Fant et al. (1991) han analizado el efecto de la acentuación en la duración de la sílaba.

9

Pointon (1978), Toledo (1988a) y Ríos (1991) hacen referencia a la posición en la unidad tonal y Klatt $(1979,1987)$ en la frase, en lugar del grupo de sentido. 10

Otros autores han analizado el efecto del tipo silábico en la duración de la sílaba, entre ellos, Gili Gaya (1940), Delattre (1966), Olsen (1972), Pointon (1978), Toledo (1988), y Ríos (1991).
} 
("Say the word", "again"), a la que se ha restado la duración de la palabra y pausas. ocasionales antes y después de la palabra objeto. Seguidamente se ha calculado la duración media de la parte fija (1066 ms.) y se han relativizado todas éstas en función de la media. Este valor, que hemos denominado coeficiente de velocidad, ha servido para dividir la duración de consonantes, vocales, sílabas y palabras, y así relativizar los datos. Sin embargo, hemos descartado aquellos casos en los que el coeficiente de velocidad resultante fue inferior a 0.7 o superior a 1.3., lo cual ha servido para corregir pequeñas oscilaciones.

Las muestras orales fueron grabadas en su totalidad en el estudio de grabación de la Facultad de Ciencias de la Información de la Universidad de Sevilla mediante una mesa de mezclas Total Audio Concepts (TAC) Scorpion II, sin ecualización, sobre una cinta DAT (Digital Audio Tape) a $48 \mathrm{Khz}$. en una platina DAT Sony DTC -57 ES, y micrófonos Sony F-730 situados a unos 24 - $30 \mathrm{cms}$. de la boca de los informantes.

El análisis acústico del corpus se realizó con un Kay DSP- Sonagraph (modelo 5500) en el Laboratorio de Fonética de la Universidad de Sevilla, que permitió la comprobación auditiva y en la onda oscilográfica de la segmentación y medición de las duraciones realizadas sobre sonogramas de banda ancha.

Para la división silábica se siguieron las normas de la lengua inglesa, tal y como se aplican en Wells (1990).

A partir de los datos gestionados por el programa informático $\mathrm{dB}$ ase, se ha procedido su tratamiento estadístico mediante los paquetes informáticos CSS: Statistica y Minitab. Se han empleado estadísticas descriptivas básicas como distribuciones de frecuencias, promedios y medidas de dispersión. También se ha recurrido al uso de correlaciones para determinar las relaciones entre variables.

No obstante, casi todo el análisis estadístico se ha basado en los análisis de varianza, ANOVA cuando se consideraba un único factor, y MANOVA en el caso de varios factores. Estos análisis son los más adecuados para determinar la incidencia de variables de tipo cualitativo, en su mayor parte dicotómicas, sobre otras de tipo cuantitativo. Entre las primeras tendremos la condición acentuada/inacentuada, la posición final/no final o el tipo silábico abierto/cerrado. Entre las segundas tendremos todas las duraciones de la silaba. Para una mayor validez de este tipo de pruebas estadísticas conviene trabajar con distribuciones normales que además presenten parámetros válidos de homogeneidad. Para comprobar estas condiciones hemos recurrido a la aplicación del test de KolmogorovSmirnov, comprobando que la mayoría de las distribuciones se ajustaban perfectamente. En cualquier caso, cuando se trabaja con un número de datos tan elevado como en estas muestras la no normalidad no influye demasiado en la validez de los resultados del ANOVA. Por la misma razón, no ha sido necesario aplicar tests específicos de homogeneidad para validar estos análisis. Para la validación de los resultados se aplica el test $\mathrm{F}$, que presenta un alto grado de robustez incluso cuando no se dan las mejores condiciones.

Finalmente, en algunas ocasiones hemos recurrido a otros tipos de técnicas como la tabulación cruzada o el test de Chi2. 


\section{La duración de la sílaba en inglés.}

El número total de sílabas en este corpus es de 1475, de las cuales 144 han respondido a la estructura silábica CV, lo que representa el 9.76\% del conjunto total de sílabas, y 406 han respondido a la estructura silábica CVC, lo que representa el $27.52 \%$ del total.

Una vez obtenidos los datos sobre duración silábica, se realizaron diversos análisis de regresión mediante los programas informáticos de análisis estadístico CSS: Statistica y Minitab, junto con distribuciones de frecuencia para cada categoría silábica.

Del análisis de casos se obtuvo para la sílaba una duración media de 234.37 ms., y la varianza 16054, con una desviación típica de $126.70 \mathrm{~ms}$. El recorrido fue de $718 \mathrm{~ms}$., con un valor mínimo de $21 \mathrm{~ms}$. y un valor máximo de $739 \mathrm{~ms}$.

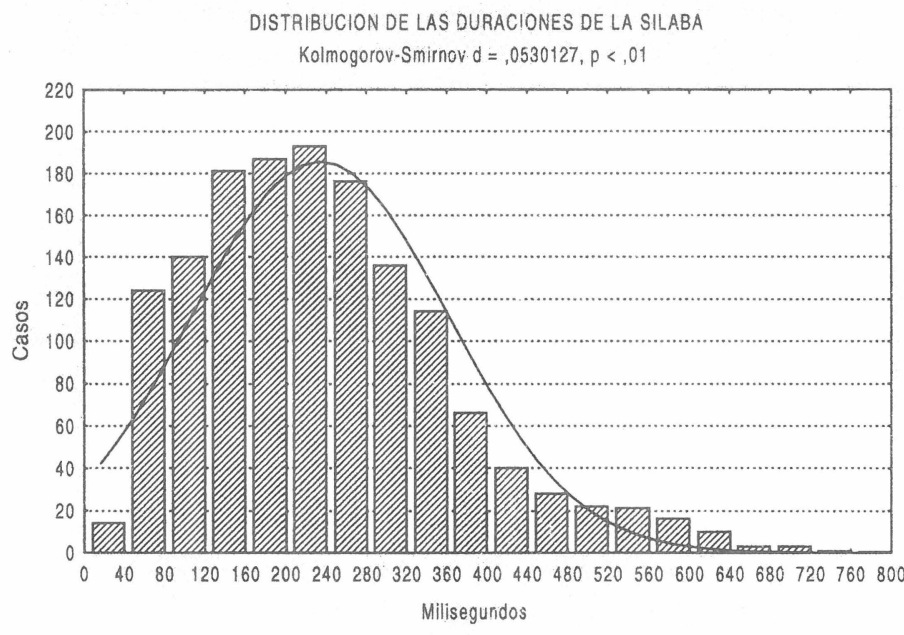

Gráfico 1

Como se aprecia en el gráfico 1, a pesar de un cierto grado de dispersión de los datos, el centro de la curva se encuentra en la clase modal (200-240 ms.) donde se sitúa la media agrupando el $13.08 \%$ de los datos, y las tres centrales el $37.68 \%$, proporcionando cierta idea de normalidad de la distribución. Por tanto, a pesar del desajuste resultante de la aplicación del test de Kolmogorov-Smirnov $(\mathrm{d}=0.053>\mathrm{dmax}=0.023)$, dadas las exigencias de este test cuando los casos son muy numerosos, podemos aplicar un análisis tipo ANOVA con el fin de averiguar qué factores y en qué medida intervienen en la duración de la sílaba, puesto que se asemeja mucho a la curva normal. De hecho, si se introdujeran menos intervalos, el desajuste desaparecería. En primer lugar, aplicamos un análisis factor a factor y los resultados obtenidos se exponen a continuación.

En el estudio de las incidencias de la condición final/no final de la sílaba en la palabra, F presentó un valor 407.736 , de manera que este factor ha resultado ser altamente 
significativo a la hora de explicar la varianza de la duración de la sílaba a un nivel de confianza superior al 99\%, dando lugar a duraciones medias para cada grupo muy dispares, tal y como refleja la tabla I.

\begin{tabular}{||l|l|l|l||}
\hline \multicolumn{4}{|c|}{ TABLA I. Caracterización sílaba en posición final/no final en la palabra } \\
\hline \hline Final/no final & Dur. Media sílaba & Desviación típica & Número de casos \\
\hline Final & 322.40 & 136.52 & 457 \\
\hline No final & 194.86 & 99.33 & 1018 \\
\hline Total & 234.37 & 126.70 & 1475 \\
\hline
\end{tabular}

Las sílabas no finales lógicamente son más numerosas que las finales y la diferencia entre las respectivas medias son muy acusadas, con desviaciones típicas igualmente dispares ${ }^{11}$. Según Klatt (1975) y Oller (1971) la función primaria de este alargamiento en inglés está relacionada con el nivel de la percepción, es decir, este ajuste temporal de alguna forma funciona como una señal de segmentación para el oyente. ${ }^{12}$

El segundo factor analizado es el de la acentuación, cuyo valor $\mathrm{F}$ ha sido 419.532, lo que quiere decir que este factor, considerado de forma aislada, ha sido muy significativo a la hora de explicar la duración de la sílaba, también a un nivel de confianza por encima del $99 \%$, como se puede apreciar comparando las duraciones medias y desviaciones típicas para los dos grupos en la tabla II.

\begin{tabular}{||l|l|l|l||}
\hline \multicolumn{4}{|c|}{ TABLA II. Caracterización sílaba acentuada/inacentuada } \\
\hline \hline Acentuada/inacentuada & Dur. Media sílaba & Desviación típica & Número de casos \\
\hline Acentuada & 323.24 & 125.44 & 458 \\
\hline Inacentuada & 194.35 & 105.11 & 1017 \\
\hline Total & 234.37 & 126.70 & 1475 \\
\hline \hline
\end{tabular}

En efecto se puede observar cómo las sílabas inacentuadas son más numerosas que las acentuadas y las duraciones medias muy dispares entre sí, claramente superiores en las

\footnotetext{
${ }^{11}$ El efecto de la posición en inglés es más relevante en este trabajo (1.65:1) que en Delattre (1966) (1.53:1).

12 Ibid. Smith (1978:44).
} 
sílabas acentuadas ${ }^{13}$, si bien la desviación típica para las sílabas inacentuadas es claramente inferior a la del conjunto general. Esto quiere decir que puede hablarse de un agrupamiento especial en esta última categoría.

El tercer factor analizado es el de tipo silábico abierto/cerrado, con un valor F 453.949, siendo, por tanto, este factor de nuevo significativo para un nivel de confianza por encima del 99\%. Por tanto, su incidencia sobre la duración de la sílaba es similar a la de los dos primeros factores analizados. Las medias obtenidas son las que se exponen en la tabla III.

\begin{tabular}{||l|l|l|l||}
\hline \multicolumn{4}{|c|}{ TABLA III. Caracterización sílaba abierta/cerrada } \\
\hline \hline Abierto / Cerrado & Dur. Media sílaba & Desviación típica & Número de casos \\
\hline Abierto & 141.44 & 84.71 & 449 \\
\hline Cerrado & 275.04 & 120.46 & 1026 \\
\hline Total & 234.37 & 126.70 & 1475 \\
\hline
\end{tabular}

Las sílabas cerradas son más numerosas y considerablemente más largas que las sílabas $\operatorname{abiertas}^{14}$, si bien la desviación típica es menor en el grupo de sílabas abiertas. La realización de la coda silábica ha supuesto una mayor duración de la sílaba debido al mayor número de segmentos que la conforman.

Con el fin de comprender mejor la influencia de estos tres factores de forma conjunta, comparando sus pesos relativos en la varianza de las variables y efectos interrelacionados, se ha recurrido al análisis multifactorial tipo MANOVA. Los resultados obtenidos son los que aparecen en la tabla IV.

13 Delattre (1966) en su estudio interlingüístico destacó que el inglés presenta unos valores superiores que el español en cuanto a la oposición entre sílabas acentuadas y sílabas inacentuadas, 1.60:1. Los resultados de este trabajo indican una oposición muy similar, 1.66:1.

14

La oposición temporal por tipo silábico fue bastante más relevante en los resultados de este estudio (1.94:1) que en Delattre (1966) (1.25:1). 


\begin{tabular}{||l|l|l|l|l|l|l||}
\hline \multicolumn{6}{|c||}{ TABLA IV. MANOVA efectos posición final/no final en la palabra, acentuación y } \\
tipo silábico para la sílaba \\
\hline \hline Effect & $\begin{array}{l}\text { df } \\
\text { Effect }\end{array}$ & $\begin{array}{l}\text { MS } \\
\text { Effect }\end{array}$ & df Error & MS Error & & p-level \\
\hline $1 *$ & 1 & 747117 & 1467 & 5739.52 & 130.170 & 0.000000 \\
$2 *$ & 1 & 1003159 & 1467 & 5739.52 & 174.780 & 0.000000 \\
$3 *$ & 1 & 62803 & 1467 & 5739.52 & 10.942 & 0.000963 \\
$12 *$ & 1 & 98096 & 1467 & 5739.52 & 17.091 & 0.000038 \\
13 & 1 & 12935 & 1467 & 5739.52 & 2.253 & 0.133508 \\
$23 *$ & 1 & 48847 & 1467 & 5739.52 & 8.510 & 0.003584 \\
123 & 1 & 727 & 1467 & 5739.52 & 0.126 & 0.721986 \\
\hline
\end{tabular}

* Estadísticamente significativo

Al igual que el análisis unifactorial, éste demuestra que todos y cada uno de los factores aisladamente explican la varianza de la duración de la sílaba, todos ellos a un nivel de confianza superior al 99\%. Asimismo, con respecto a los efectos combinados, únicamente las interrelaciones entre la acentuación y los otros dos factores cobran significación. En estos tres factores obtenemos grupos distintos con las duraciones medias y desviaciones típicas que aparecen en la tabla V.

Los grupos finales en general presentan las duraciones medias más elevadas, sobre todo el grupo de sílabas "final acentuada cerrada". Por el contrario el valor mínimo fue presentado por el grupo de sílabas "no final inacentuada abierta". En todos los grupos las desviaciones típicas decrecen considerablemente respecto a la general, lo que quiere decir que puede hablarse de compartimientos más homogéneos dentro de cada grupo.

Con el objeto de analizar la influencia de otros factores sobre la duración de la sílaba, a los que no convenía aplicar un análisis tipo ANOVA por el excesivo número de categorías, en la tabla VI se han calculado las correlaciones. 


\begin{tabular}{||l|l|l|l||}
\hline \multicolumn{3}{|c||}{$\begin{array}{l}\text { TABLA V. Caracterización sílaba en posición final/no final en la palabra, } \\
\text { acentuada/inacentuada y abierta/cerrada }\end{array}$} \\
\hline \hline $\begin{array}{l}\text { Final/no final Acentuada/inacentuada } \\
\text { Abierta/cerrada }\end{array}$ & $\begin{array}{l}\text { Dur. Media } \\
\text { sílaba }\end{array}$ & $\begin{array}{l}\text { Desviación } \\
\text { típica }\end{array}$ & $N^{\circ}$ \\
\hline Final Acentuada Abierta & 502.12 & 46.93 & 4 \\
Final Acentuada Cerrada & 524.96 & 89.46 & 82 \\
Final Inacentuada Abierta & 195.34 & 62.84 & 123 \\
Final Inacentuada Cerrada & 315.54 & 87.02 & 248 \\
No final Acentuada Abierta & 287.99 & 71.59 & 3 \\
No final Acentuada Cerrada & 276.76 & 78.38 & 369 \\
No final Inacentuada Abierta & 114.76 & 69.75 & 319 \\
No final Inacentuada Cerrada & 179.72 & 69.92 & 327 \\
\hline Todos los grupos & 234.37 & 126.70 & 1475 \\
\hline
\end{tabular}

\begin{tabular}{||l|l||}
\hline \multicolumn{2}{|c|}{ TABLA VI. Correlaciones para la sílaba } \\
\hline$N^{\circ}$ sílabas en la palabra & -0.3892 \\
\hline$N^{\circ}$ fonos en la palabra & -0.2823 \\
\hline Posición respecto al acento & 0.1637 \\
\hline Posición final/no final en palabra & 0.4673 \\
\hline Tipo abierto/cerrado & 0.4842 \\
\hline Acentuación & 0.4701 \\
\hline$N^{\circ}$ fonos en la sílaba & 0.6771 \\
\hline
\end{tabular}

Es especialmente fuerte la correlación entre el número de fonos que conforman la sílaba y la duración de la sílaba, de manera que cuanto mayor es el número de fonos en la sílaba, mayor es su duración, tal y como se aprecia en el gráfico 2.

15 Unicamente aparecen las correlaciones significativas. 


\section{DURACIÓN DE LA SÍLABA SEGÚN EL NÚMERO DE FONOS QUE LA COMPONEN}

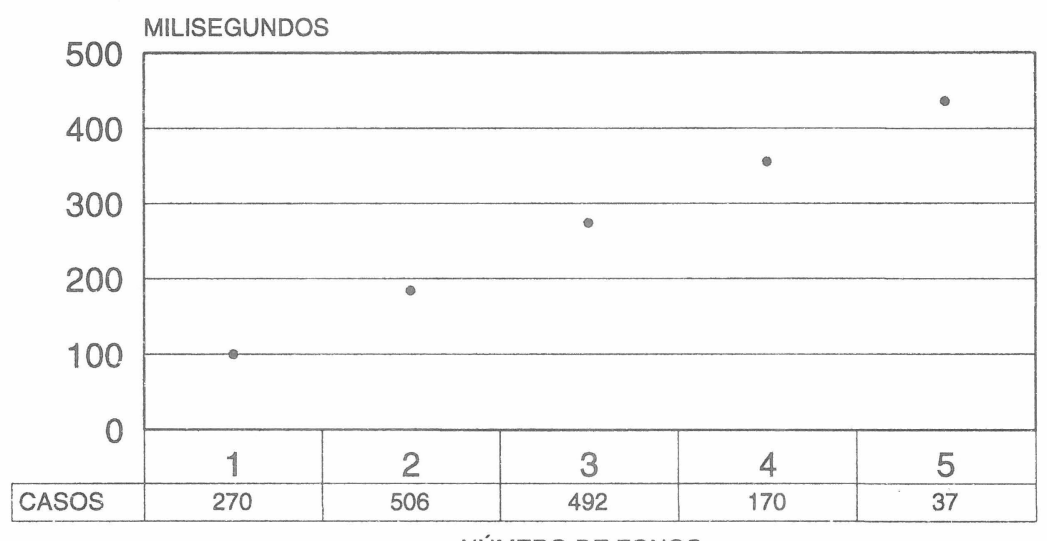

NÚMERO DE FONOS

Gráfico 2

También significativas, aunque de menor peso, han sido las correlaciones negativas entre la duración de la sílaba y el número de fonos que conforman la palabra, o entre la duración de la sílaba y el número de sílabas que conforman la palabra.

\section{DURACIÓN DE LA SÍLABA SEGÚN EL NÚMERO DE FONOS QUE CONFORMAN LA PALABRA}

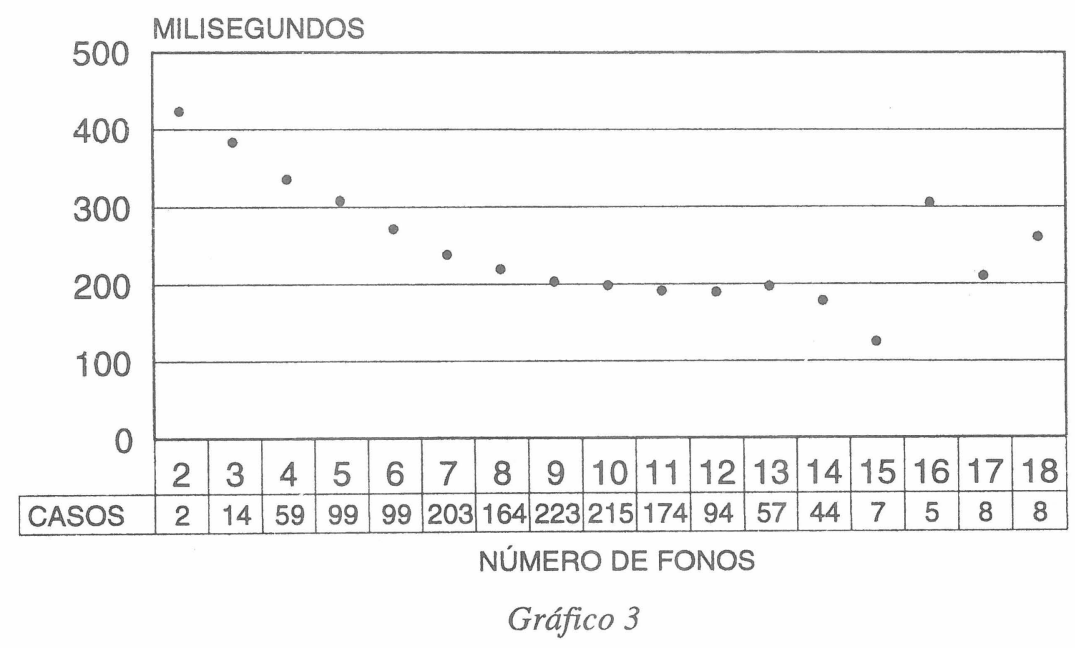


Como se aprecia en los gráficos 3 y 4, a medida que aumenta el número de fonos o sílabas en la palabra, tiende a decrecer la duración de la sílaba.

\section{DURACIÓN DE LA SÍLABA SEGÚN EL NÚMERO DE SÍLABAS QUE CONFORMAN LA PALABRA}

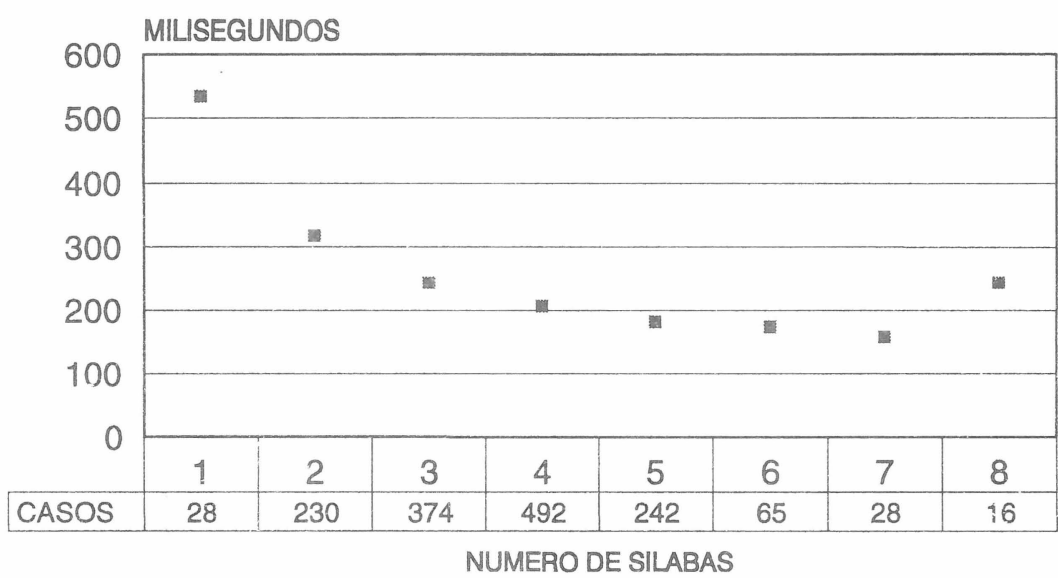

Gráfico 4

En el gráfico 5 se observa que el comportamiento de la sílaba en función de la posición respecto a la sílaba acentuada fue más bien errático, no siendo nada clara su correlación. No obstante, se observa una tendencia a un patrón rítmico alternante en la sucesión de sílabas átonas en la palabra.

Las 440 sílabas preacentuales presentaron una duración media de 157.95 ms. y una desviación típica de 81.51 ms., mientras que las 578 sílabas postacentuales presentaron una duración media de $223.40 \mathrm{~ms}$. y una desviación típica de $42.61 \mathrm{~ms}$. Por tanto, las sílabas postacentuales, en general, son más largas que las preacentuales, y se aprecia un mayor agrupamiento en sus datos. No obstante, es la sílaba acentuada la que presenta un valor medio de duración mayor. Las 457 sílabas acentuadas presentaron un valor medio de $321.83 \mathrm{~ms}$. y una desviación típica de $125.94 \mathrm{~ms}$.

Ese alargamiento de la duración de la sílaba acentuada se confirma también si tan sólo tenemos en cuenta sílabas no finales en nuestro análisis, de cuyo análisis de casos se obtuvo para la sílaba una duración media de 194.86 ms. y una desviación típica de $99.33 \mathrm{~ms}$. El recorrido fue de $554 \mathrm{~ms}$., con un valor mínimo de $21 \mathrm{~ms}$. y un valor máximo de $575 \mathrm{~ms}$.

Por otra parte, nada significativa ha sido la correlación entre la duración de la sílaba y la duración de la palabra. 


\section{DURACION DE LA SILABA SEGUN LA POSICION RESPECTO AL ACENTO}

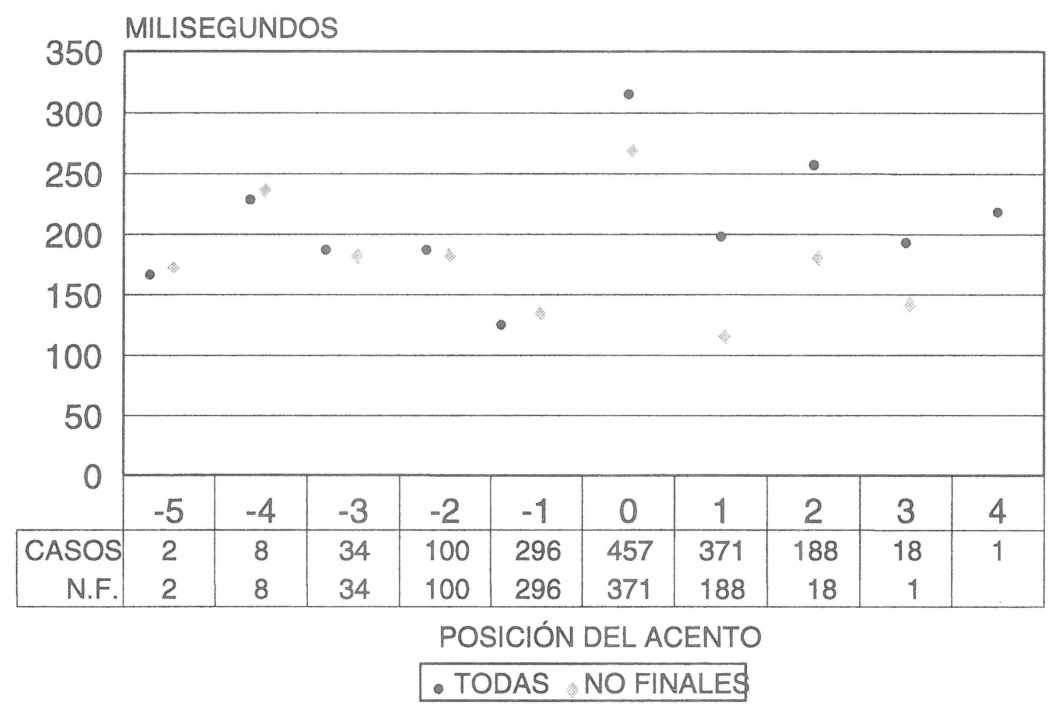

Gráfico 5

Finalmente, se ha llevado a cabo un análisis de la varianza para evaluar la influencia de la estructura silábica en la duración de la sílaba. Para ello se compararon únicamente las estructuras silábicas con el mismo número de segmentos: un primer grupo lo formaron $\mathrm{CV}$ y VC, un segundo grupo $\mathrm{VCC}, \mathrm{CVC}$ y $\mathrm{CCV}$, y finalmente, un tercer grupo estuvo compuesto por CCVC y $\mathrm{CVCC}^{16}$. A todos ellos se ha aplicado un análisis tipo ANOVA, cuyo valor $\mathrm{F}$ fue 1.01983 , es decir, este factor no ha resultado ser significativo a la hora de explicar la variabilidad en la duración de la sílaba, si bien la estructura silábica VC fue ligeramente más larga, además de considerablemente más numerosa, acorde con la tendencia general del sistema lingüístico bajo estudio, tal y como se aprecia en la tabla VII.

El valor de F para el segundo grupo fue 17.6496, siendo, por tanto, un factor altamente significativo para este segundo grupo de sílabas, por encima de un nivel de confianza del 99\%, donde las estructuras silábicas cerradas resultaron más largas, con desviaciones típicas inferiores a la del conjunto total de sílabas, lo que quiere decir que puede hablarse de agrupamientos especiales para cada grupo, como se ve en la tabla VIII.

16 La estructura silábica V, con 270 casos, presentó un valor medio de 109.70 ms. y una desviación típica de 63.79 ms. 


\begin{tabular}{||l|l|l|l||}
\hline \multicolumn{3}{|c|}{ TABLA VII. Caracterización sílaba (2 segmentos) según la estructura silábica } \\
\hline \hline Estructura silábica & $\begin{array}{l}\text { Duración media } \\
\text { sílaba }\end{array}$ & Desviación típica & Número de casos \\
\hline CV & 187.56 & 81.60 & 144 \\
\hline VC & 195.68 & 81.59 & 362 \\
\hline
\end{tabular}

\begin{tabular}{||l|l|l|l||}
\hline \multicolumn{4}{|c||}{ TABLA VIII. Caracterización sílaba (3 segmentos) según la estructura silábica } \\
\hline \hline Estructura silábica & $\begin{array}{l}\text { Duración media } \\
\text { sílaba }\end{array}$ & Desviación típica & Número de casos \\
\hline VCC & 293.29 & 83.67 & 51 \\
\hline CVC & 290.96 & 104.60 & 406 \\
\hline CCV & 185.95 & 103.20 & 36 \\
\hline
\end{tabular}

Sin embargo, no hemos aplicado ANOVA al tercer y cuarto grupos debido al escaso número de casos, sobre todo en sílaba abierta. Los datos de las tablas IX y X deben, por consiguiente, ser considerados meros indicadores de algunas tendencias.

\begin{tabular}{||l|l|l|l||}
\hline \multicolumn{4}{||c||}{ TABLA IX. Caracterización sílaba (4 segmentos) según la estructura silábica } \\
\hline \hline Estructura silábica & Duración media sílaba & Desviación típica & Número de casos \\
\hline CCVC & 405.85 & 123.23 & 68 \\
\hline CVCC & 380.80 & 112.53 & 96 \\
\hline VCCC & 394.39 & 38.58 & 4 \\
\hline CCCV & 539.99 & 0 & 1 \\
\hline
\end{tabular}




\begin{tabular}{||l|l|l|l||}
\hline \multicolumn{4}{|c|}{ TABLA X. Caracterización sílaba (5 segmentos) según la estructura silábica } \\
\hline \hline $\begin{array}{l}\text { Estructura } \\
\text { silábica }\end{array}$ & $\begin{array}{l}\text { Duración media } \\
\text { sílaba }\end{array}$ & Desviación típica & Número de casos \\
\hline CCVCC & 453.66 & 122.63 & 26 \\
\hline CCCVC & 405.85 & 112.24 & 8 \\
\hline CVCCC & 481.93 & 131.61 & 3 \\
\hline
\end{tabular}

Creemos más apropiado considerar este factor no estadísticamente significativo, puesto que tan sólo aparece como tal para el segundo grupo, y puede ser debido a otros factores como la posición final/no final o la acentuación. No obstante, ha sido obvia la mayor duración de la sílaba conforme a la mayor complejidad estructural. Esto puede en realidad explicarse por un mayor número de segmentos en la sílaba, de ahí que hayamos aplicado una tabulación cruzada en la tabla XI.

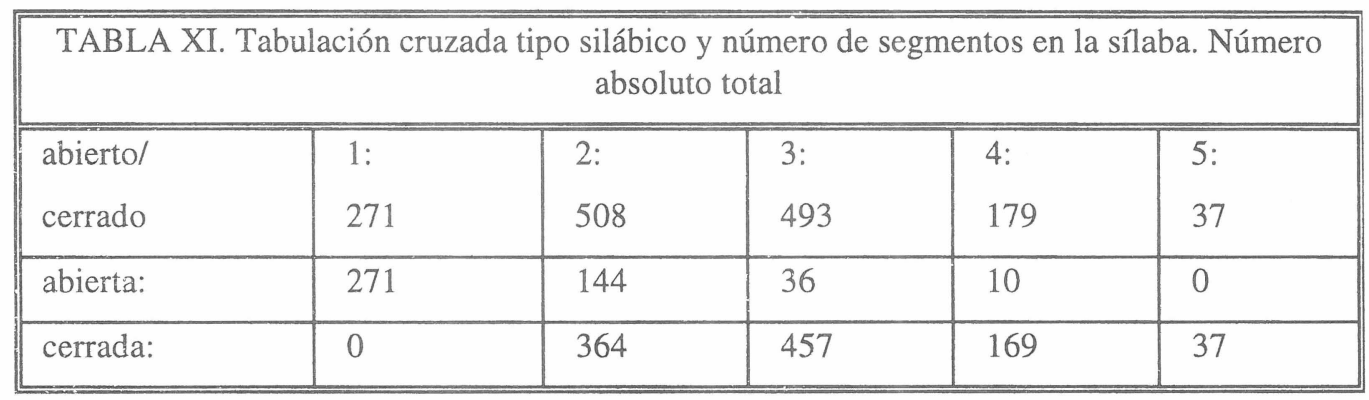

Se puede comprobar cómo el $92 \%$ de sílabas abiertas están formadas por 1 ó 2 segmentos, mientras que lógicamente no se da caso alguno de sílaba cerrada con un único segmento, y las sílabas cerradas de dos segmentos representan tan sólo el 35.4\% del total de sílabas cerradas. Por el contrario, el $60.9 \%$ de las sílabas cerradas están formadas por 3 ó 4 segmentos, frente al $8.2 \%$ de las sílabas abiertas.

Es indiscutible el predominio de la estructura silábica cerrada en inglés. En este corpus el $69.55 \%$ de las sílabas responden al tipo silábico cerrado, lo cual concuerda con el $60 \%$ señalado por Delattre (1965:42). En la tabla XII se indica la incidencia de las estructuras silábicas más frecuentes en inglés en este trabajo, en Dauer (1983:56) y en Delattre (1965:41). 


\begin{tabular}{||l|l|l|l||}
\hline \multicolumn{4}{||c|}{ TABLA XII. Incidencia de las estructuras silábicas más frecuentes en inglés. \% } \\
\hline \hline Estructura & Este trabajo & Dauer (1983) & Delattre (1965) \\
\hline V & 18.30 & 8 & -- \\
\hline CV & 9.76 & 34 & 27.6 \\
\hline VC & 24.54 & 15 & 11.9 \\
\hline CVC & 27.52 & 30 & 31.8 \\
\hline CCV & 2.44 & -- & 4 \\
\hline CVCC & 6.50 & 6 & -- \\
\hline \hline
\end{tabular}

Como puede apreciarse, el tipo silábico con mayor número de casos en este corpus ha sido CVC, lo cual coincide con Delattre (1965), mientras que el tipo silábico con mayor número de casos en Dauer (1983) ha sido CV.

Asimismo, en el gráfico 6 se ha aplicado una tabulación cruzada con el fin de comprobar el mayor porcentaje de sílabas acentuadas conforme a la mayor complejidad estructural.

\section{ESTRUCTURA SILABICA Y ACENTUACION}

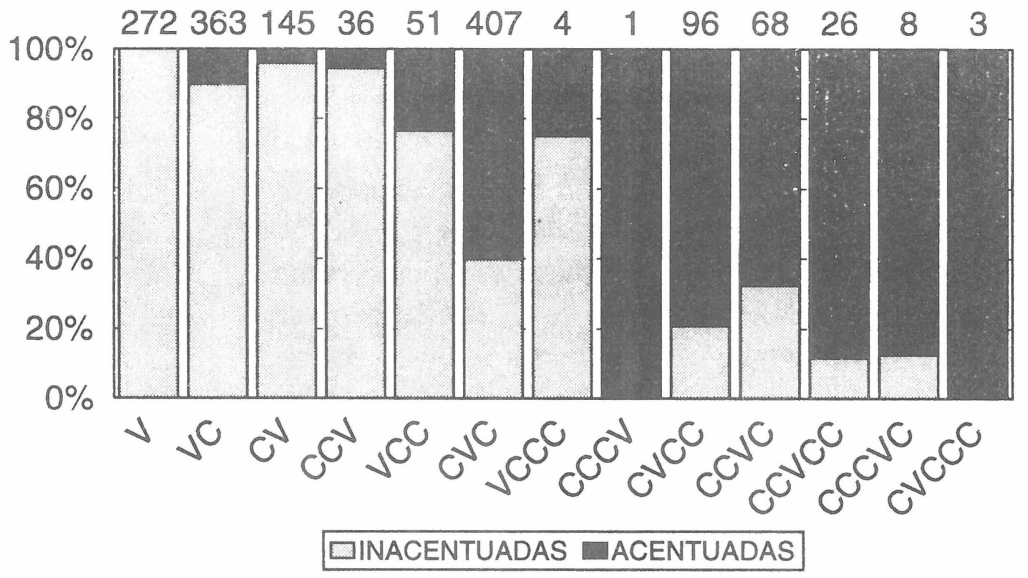

Gráfico 6 
Igualmente hemos comprobado la interrelación existente entre acentuación y complejidad silábica en inglés al aplicar el test de Chi2 en la tabla XIII. Se han ignorado las estructuras VCCC, CCCV, CVCCC y CCCVC, al contar con tan sólo 4, 1, 3 y 8 datos respectivamente, lo cual los hace poco significativos y al mismo tiempo invalidaría el test de Chi2.

\begin{tabular}{||l|l|l|l|l}
\hline \hline \multicolumn{4}{c}{ TABLA XIII. Chi 2 acentuación y estructura silábica para la sílaba } \\
\hline \hline \multirow{2}{*}{ Estructura silábica } & \multicolumn{2}{|c|}{ Sílabas Acentuadas } & \multicolumn{2}{l}{ Sílabas Inacentuadas } \\
\cline { 2 - 5 } & $\begin{array}{l}\text { Valores } \\
\text { observados }\end{array}$ & $\begin{array}{l}\text { Valores } \\
\text { esperados }\end{array}$ & $\begin{array}{l}\text { Valores } \\
\text { observados }\end{array}$ & $\begin{array}{l}\text { Valores } \\
\text { esperados }\end{array}$ \\
\hline CVC & 245 & 126 & 162 & 281 \\
CV & 6 & 45 & 139 & 100 \\
VC & 37 & 113 & 326 & 250 \\
CCV & 2 & 11 & 34 & 25 \\
VCC & 12 & 16 & 39 & 35 \\
V & 0 & 84 & 272 & 188 \\
CCVC & 46 & 21 & 2220 & 47 \\
CVCC & 76 & 30 & 3 & 66 \\
CCVCC & 23 & 8 & & 18 \\
\hline \hline
\end{tabular}

Chi2 $=605 ; \mathrm{gl}=8 ; \mathrm{p}=0$

Por tanto, se confirma que en general las sílabas más complejas serán acentuadas, frente a las estructuras silábicas más simples, que en su mayoría serán inacentuadas. Esto concuerda con los resultados de Dauer $(1983: 56)^{17}$ o Fant et al. (1991c:352): una media de 3.1 fonemas en sílabas acentuadas y 2.3 fonemas en sílabas inacentuadas.

\section{Conclusiones.}

La mayoría de los factores analizados, con excepción de la duración de la palabra y la estructura silábica, han sido significativos para la duración de la sílaba en inglés, en orden de importancia: el número de fonos que conforman la sílaba, el tipo silábico

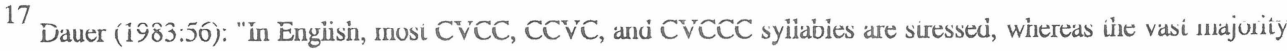
of $\mathrm{V}$ and CV syllables are unstressed."
} 
abierto/cerrado, la acentuación, la posición final/no final en la palabra, el número de sílabas que conforman la palabra, el número de fonos que conforman la palabra y, por último, la posición respecto a la sílaba acentuada. Por consiguiente, la duración de la sílaba aumenta con el incremento del número de fonos. Asimismo, son más largas las sílabas cerradas que las abiertas, las acentuadas que las inacentuadas, en posición final de palabra que en posición no final, y en posición postacentual que en posición preacentual, aunque sin alcanzar la duración de la sílaba acentuada. ${ }^{18}$ Las sílabas preacentuales han crecido en las siguientes proporciones conforme se alejan del acento: $0.43: 1,0.58: 1,0.58: 1,0.75: 1$, y $0.55: 1$, frente a las sílabas postacentuales: $0.63: 1,0.81: 1,0.62: 1$, y $0.69: 1$, revelando la presencia de un patrón alternante en la sucesión de sílabas átonas en la palabra en inglés R.P.

En cambio, la duración de la sílaba tiende a decrecer con el incremento del número de fonos o sílabas en la palabra, claros indicios de compresión intersilábica, frente a la falta de compensación intrasilábica. Es decir, las características fonéticas del inglés R.P. responderían a las de una lengua rítmicamente clasificada como de compás acentual.

Asimismo, se ha constatado una notable variedad de tipos silábicos, así como una interrelación entre acentuación y complejidad silábica, de manera que las sílabas más complejas tienden a ser acentuadas, frente a las sílabas más simples, que tienden a ser inacentuadas. Por consiguiente, las características fonológicas del inglés y los procesos que provocan acrecientan las diferencias entre las sílabas acentuadas y las sílabas inacentuadas, y regularizan la recurrencia del golpe de acento.

En conclusión, en la orientación escalar adoptada las características fonéticas y fonológicas de la sílaba en inglés aproximan al inglés R.P. al patrón de una lengua de compás acentual.

\section{Bibliografía}

Bertinetto, P.M. "Reflections on the Dichotomy 'Stress' vs. 'Syllable- timing", Revue de Phonetique Appliquée 91-92-93 (1989), 99-127

Cantín, M. y Ríos, A. "Análisis experimental del ritmo de la lengua catalana”, ASJU XXV-2 (1991), 487-514

Couper-Kuhlen, E. An Introduction to English Prosody (London 1986)

Dauer, R.M. "Stress-timing and Syllable-timing Reanalyzed", Journal of Phonetics 11 (1983), 51-62

"Phonetic and Phonological Components of Language Rhythm", Proceedings of the Xith International Congress of Phonetic Sciences 5 (1987), 447-450

\footnotetext{
18 Estos resultados confirman la opinión de Nooteboom (1991:233): "The pattern shown correctly predicts of course, that the lexically stressed syllable is most prominent, and has the longest syllable duration."
} 
Delattre, P. "Comparing the Prosodic Features of English, German, Spanish and French", Phonetic Features (Heidelberg 1965)

"A Comparison of Syllable Length Conditioning among Languages" International Review of Applied Linguistics 4 (1966), 183-198

Engstrand, O. "Duration Patterns of Lule Sami Phonology" Phonetica 44 (1987), 117 128

Fant, G., Kruckenberg, A. \& Nord, L. "Durational Correlates of Stress in Swedish, French and English" Journal of Phonetics 19 (1991), 351-365

Fauré, G., Hirst, D.H., \& Chaufcouloff, M. "Rhythm in English: Isochronism, Pitch and Perceived Stress" The Melody of Language. Intonation and Prosody (Baltimore 1980)

Fowler, C.A. Timing Control in Speech Production (Bloomington 1977)

Giegerich, H.J. Metrical phonology and phonological structure: German and English (Cambridgge 1985) English Phonology (Cambridge 1992)

Gili Gaya, S. "La cantidad silábica en la frase" Castilla 1 (1940), 287-298

Gutiérrez, F., Monroy, R. y Schew, D. "El aprendizaje del ritmo inglés por hispanohablantes: bases fonéticas" Actas del VIII Congreso Nacional AESLA (Vigo 1992), 349-359

Hoequist, C.E. "Durational Correlates of Linguistic Rhythm Categories" Phonetica 40 (1983a), 19-31

"Syllable Duration in Stress-, Syllable- and Mora-timed Languages" Phonetica 40 (1983b), 203-237

Kiatt, D.H. "Synthesis by Rule of Segmental Durations in English Sentences" Frontiers of Speech Communication Research (London 1979)

"Review of Text-to-Speech Conversion for English" Journal of the Acoustical Society of America 82 (1987), 460-464

Lindblom, B.E.F. "Some Temporal Regularities of Spoken Swedish" Auditory Analysis and Perception of Speech (London 1975), 387-396

Macarrón, A., Escalada, G. \& Rodríguez, M.A. "Generation of Duration Rules for a Spanish Text-to-Speech Synthesizer" EUROSPEECH (1991), 617-620

Nakatani, L.H., O'Connor, K.D. \& Aston, C.H. "Prosodic Aspects of American English Speech Rhythm" Phonetica 38 (1981), 84-106

Pike, K.L. The Intonation of American English (Michigan 1945) 
